\title{
Positive solutions for Hadamard fractional differential equations on infinite domain
}

\author{
Phollakrit Thiramanus ${ }^{1}$, Sotiris K Ntouyas ${ }^{2,3}$ and Jessada Tariboon ${ }^{1,4^{*}}$
}

\author{
"Correspondence: \\ jessada.t@sci.kmutnb.ac.th \\ ${ }^{1}$ Nonlinear Dynamic Analysis \\ Research Center, Department of \\ Mathematics, Faculty of Applied \\ Science, King Mongkut's University \\ of Technology North Bangkok, \\ Bangkok, 10800, Thailand \\ ${ }^{4}$ Centre of Excellence in \\ Mathematics, CHE, Sri Ayutthaya \\ Rd., Bangkok, 10400, Thailand \\ Full list of author information is \\ available at the end of the article
}

\begin{abstract}
This paper investigates the existence of nonnegative multiple solutions for nonlinear fractional differential equations of Hadamard type, with nonlocal fractional integral boundary conditions on an unbounded domain by means of Leggett-Williams and Guo-Krasnoselskii's fixed point theorems. Two examples are discussed for illustration of the main work.
\end{abstract}

MSC: 34A08; 34A12; 34B15; 34B40

Keywords: Hadamard fractional derivative; Hadamard fractional integral; fractional integral boundary conditions; fixed point theorems

\section{Introduction}

Fractional calculus has gained considerable attention from both theoretical and the applied points of view in recent years. There are numerous applications in a variety of fields such as electrical networks, chemical physics, fluid flow, economics, signal and image processing, viscoelasticity, porous media, aerodynamics, modeling for physical phenomena exhibiting anomalous diffusion, and so on. In contrast to integer-order differential and integral operators, fractional-order differential operators are nonlocal in nature and provide the means to look into hereditary properties of several materials and processes. The monographs [1-4] are commonly cited for the theory of fractional derivatives and integrals and applications to differential equations of fractional order. For more details and examples, see [5-10] and the references therein.

However, it has been noticed that most of the work on the topic is concerned with Riemann-Liouville or Caputo type fractional differential equations. Besides these fractional derivatives, another kind of fractional derivatives found in the literature is the fractional derivative due to Hadamard introduced in 1892 [11], which differs from the aforementioned derivatives in the sense that the kernel of the integral in the definition of Hadamard derivative contains logarithmic function of arbitrary exponent. A detailed description of Hadamard fractional derivative and integral can be found in [1,12-18].

Boundary value problems on infinite intervals appear often in applied mathematics and physics, such as in unsteady flow of gas through a semi-infinite porous medium, the theory of drain flows, etc. More examples and a collection of works on the existence of solutions of boundary value problems on infinite intervals for differential, difference and integral 
equations may be found in the monographs $[19,20]$. For boundary value problems of fractional order on infinite intervals we refer to [21-25].

Zhao and Ge [25] studied the existence of unbounded solutions for the following boundary value problem on the infinite interval:

$$
\begin{aligned}
& D_{0}^{\alpha} u(t)+f(t, u(t))=0, \quad 1<\alpha \leq 2, t \in[0, \infty), \\
& u(0)=0, \quad \lim _{t \rightarrow \infty} D_{0}^{\alpha-1} u(t)=\beta u(\xi),
\end{aligned}
$$

where $D_{0}^{\alpha}$ denotes Riemann-Liouville fractional derivative of order $\alpha$, and $0<\beta, \xi<\infty$.

Zhang et al. [24] studied the existence of nonnegative solutions for the following boundary value problem for fractional differential equations with nonlocal boundary conditions on unbounded domains:

$$
\begin{aligned}
& D_{0}^{\alpha} u(t)+f(t, u(t))=0, \quad 1<\alpha \leq 2, t \in[0, \infty), \\
& I_{0}^{\alpha-2} u(0)=0, \quad \lim _{t \rightarrow \infty} D_{0}^{\alpha-1} u(t)=\beta I_{0}^{\alpha-1} u(\eta),
\end{aligned}
$$

where $D_{0}^{\alpha}$ denotes Riemann-Liouville fractional derivative of order $\alpha, f \in C([0, \infty) \times$ $\mathbb{R}, \mathbb{R}^{+}$) and $0<\beta, \eta<\infty$. The Leray-Schauder nonlinear alternative is used.

Liang and Zhang [22] used a fixed-point theorem for operators on a cone, proved the existence of positive solutions to the following fractional boundary value problem:

$$
\begin{aligned}
& D_{0}^{\alpha} u(t)+f(t, u(t))=0, \quad 2<\alpha \leq 3, t \in[0, \infty), \\
& u(0)=u^{\prime}(0)=0, \quad \lim _{t \rightarrow \infty} D_{0}^{\alpha-1} u(t)=\sum_{i=1}^{m-2} \beta_{i} u\left(\xi_{i}\right),
\end{aligned}
$$

where $D_{0}^{\alpha}$ denotes Riemann-Liouville fractional derivative of order $\alpha, f \in C([0, \infty) \times$ $\left.\mathbb{R}, \mathbb{R}^{+}\right), 0<\xi_{1}<\xi_{2}<\cdots<\xi_{m-2}<\infty, \beta_{i} \geq 0, i=1,2, \ldots, m-2$, with $0<\sum_{i=1}^{m-2} \beta_{i} \xi_{i}^{\alpha-1}<\Gamma(\alpha)$.

Recently in [26] we studied a new class of boundary value problems on fractional differential equations with $m$-point Erdélyi-Kober fractional integral boundary conditions on an infinite interval of the form

$$
\begin{aligned}
& D_{0}^{\alpha} u(t)+f(t, u(t))=0, \quad 1<\alpha \leq 2, t \in(0, \infty), \\
& u(0)=0, \quad D_{0}^{\alpha-1} u(\infty)=\sum_{i=1}^{m-2} \beta_{i} I_{\eta_{i}}^{\gamma_{i}, \delta_{i}} u\left(\xi_{i}\right),
\end{aligned}
$$

where $D_{0}^{\alpha}$ denotes the Riemann-Liouville fractional derivative of order $\alpha, I_{\eta_{i}}^{\gamma_{i}, \delta_{i}}$ is the Erdélyi-Kober fractional integral of order $\delta_{i}>0$ with $\eta_{i}>0, \gamma_{i} \in \mathbb{R}, i=1,2, \ldots, m-2$, $\beta_{i} \in \mathbb{R}$, and $\xi_{i} \in(0, \infty), i=1,2, \ldots, m-2$ are given constants. We proved the existence and uniqueness of unbounded solution of the boundary value problem (1.9)-(1.10) by using the Leray-Schauder nonlinear alternative and Banach contraction principle.

In this paper, we aim to investigate the existence criteria of positive solutions for fractional differential equations of Hadamard type, with integral boundary condition on infinite intervals. Precisely, we consider the following boundary value problem for Hadamard 
fractional differential equations:

$$
\begin{aligned}
& D^{\alpha} u(t)+a(t) f(u(t))=0, \quad 1<\alpha \leq 2, t \in(1, \infty), \\
& u(1)=0, \quad D^{\alpha-1} u(\infty)=\sum_{i=1}^{m} \lambda_{i} I^{\beta_{i}} u(\eta)
\end{aligned}
$$

where $D^{\alpha}$ denotes the Hadamard fractional derivative of order $\alpha, \eta \in(1, \infty)$, and $I^{\beta_{i}}$ is the Hadamard fractional integral of order $\beta_{i}>0, i=1,2, \ldots, m$, and $\lambda_{i} \geq 0, i=1,2, \ldots, m$ are given constants.

To the best of our knowledge, there are no papers devoted to the study of positive solutions for Hadamard fractional differential equations on infinite intervals; we fill the gap in this paper. As we know, $[1, \infty)$ is noncompact. In the literature a special Banach space were introduced. Unfortunately this Banach space is not suitable for Hadamard fractional differential equations. In order to overcome this difficulty, a special Banach space is introduced so that we can establish some inequalities, which guarantee that the functionals defined on $[1, \infty)$ have better properties. Applying first the well-known Leggett-Williams fixed point theorem, we obtain a new result on the existence of at least three distinct nonnegative solutions under some conditions. Next we prove the existence of at least one positive solution by using Guo-Krasnoselskii's fixed point theorem.

The rest of the paper is organized as follows: In Section 2, we present some preliminaries and lemmas that will be used to prove our main results. We also obtain the corresponding Green's function and some of its properties. The main result is formulated and proved in Sections 3 and 4. Especially in Section 3 we prove the existence of at least three distinct nonnegative solutions while, in Section 4, we prove the existence of at least one positive solution. Examples illustrating our results are presented in Section 5.

\section{Preliminaries}

In this section, we introduce some notations and definitions of fractional calculus [1] and present preliminary results needed in our proofs later.

Definition 2.1 [1] The Hadamard fractional integral of order $q$ for a function $g$ is defined as

$$
I^{q} g(t)=\frac{1}{\Gamma(q)} \int_{1}^{t}\left(\log \frac{t}{s}\right)^{q-1} \frac{g(s)}{s} d s, \quad q>0,
$$

provided the integral exists.

Definition 2.2 [1] The Hadamard derivative of fractional order $q$ for a function $g$ : $[1, \infty) \rightarrow \mathbb{R}$ is defined as

$$
D^{q} g(t)=\frac{1}{\Gamma(n-q)}\left(t \frac{d}{d t}\right)^{n} \int_{1}^{t}\left(\log \frac{t}{s}\right)^{n-q-1} \frac{g(s)}{s} d s, \quad n-1<q<n, n=[q]+1,
$$

where $[q]$ denotes the integer part of the real number $q$ and $\log (\cdot)=\log _{e}(\cdot)$. 
Lemma 2.3 ([1], Property 2.24) If $a, \alpha, \beta>0$ then

$$
\left(D_{a}^{\alpha}\left(\log \frac{t}{a}\right)^{\beta-1}\right)(x)=\frac{\Gamma(\beta)}{\Gamma(\beta-\alpha)}\left(\log \frac{x}{a}\right)^{\beta-\alpha-1} .
$$

Lemma 2.4 $[1,2]$ Let $q>0$ and $x \in C[1, \infty) \cap L^{1}[1, \infty)$. Then the Hadamard fractional differential equation $D^{q} x(t)=0$ has the solutions

$$
x(t)=\sum_{i=1}^{n} c_{i}(\log t)^{q-i},
$$

and the following formula holds:

$$
I^{q} D^{q} x(t)=x(t)+\sum_{i=1}^{n} c_{i}(\log t)^{q-i}
$$

where $c_{i} \in \mathbb{R}, i=1,2, \ldots, n$, and $n-1<q<n$.

Lemma 2.5 Let $h \in C[1, \infty)$ with $0<\int_{1}^{\infty} h(s) \frac{d s}{s}<\infty$, and

$$
\Omega=\Gamma(\alpha)-\sum_{i=1}^{m} \frac{\lambda_{i} \Gamma(\alpha)}{\Gamma\left(\alpha+\beta_{i}\right)}(\log \eta)^{\alpha+\beta_{i}-1}>0
$$

Then the unique solution of the following fractional differential equation:

$$
D^{\alpha} u(t)+h(t)=0, \quad t \in(1, \infty), \alpha \in(1,2),
$$

subject to the boundary conditions

$$
u(1)=0, \quad D^{\alpha-1} u(\infty)=\sum_{i=1}^{m} \lambda_{i} I^{\beta_{i}} u(\eta)
$$

is given by the integral equation

$$
u(t)=\int_{1}^{\infty} G(t, s) h(s) \frac{d s}{s},
$$

where

$$
G(t, s)=g(t, s)+\sum_{i=1}^{m} \frac{\lambda_{i}(\log t)^{\alpha-1}}{\Omega \Gamma\left(\alpha+\beta_{i}\right)} g_{i}(\eta, s)
$$

and

$$
\begin{aligned}
& g(t, s)=\frac{1}{\Gamma(\alpha)} \begin{cases}(\log t)^{\alpha-1}-\left(\log \frac{t}{s}\right)^{\alpha-1}, & 1 \leq s \leq t<\infty, \\
(\log t)^{\alpha-1}, & 1 \leq t \leq s<\infty,\end{cases} \\
& g_{i}(\eta, s)= \begin{cases}(\log \eta)^{\alpha+\beta_{i}-1}-\left(\log \frac{\eta}{s}\right)^{\alpha+\beta_{i}-1}, & 1 \leq s \leq \eta<\infty, \\
(\log \eta)^{\alpha+\beta_{i}-1}, & 1 \leq \eta \leq s<\infty .\end{cases}
\end{aligned}
$$


Proof Applying the Hadamard fractional integral of order $\alpha$ to both sides of (2.2), we have

$$
u(t)=c_{1}(\log t)^{\alpha-1}+c_{2}(\log t)^{\alpha-2}-\frac{1}{\Gamma(\alpha)} \int_{1}^{t}\left(\log \frac{t}{s}\right)^{\alpha-1} h(s) \frac{d s}{s},
$$

where $c_{1}, c_{2} \in \mathbb{R}$.

The first condition of (2.3) implies $c_{2}=0$. Therefore,

$$
u(t)=c_{1}(\log t)^{\alpha-1}-\frac{1}{\Gamma(\alpha)} \int_{1}^{t}\left(\log \frac{t}{s}\right)^{\alpha-1} h(s) \frac{d s}{s} .
$$

In accordance with Lemma 2.3, we have

$$
D^{\alpha-1} u(t)=c_{1} \Gamma(\alpha)-\int_{1}^{t} h(s) \frac{d s}{s} .
$$

The second condition of (2.3) leads to

$$
c_{1}=\frac{1}{\Omega}\left(\int_{1}^{\infty} h(s) \frac{d s}{s}-\sum_{i=1}^{m} \frac{\lambda_{i}}{\Gamma\left(\alpha+\beta_{i}\right)} \int_{1}^{\eta}\left(\log \frac{\eta}{s}\right)^{\alpha+\beta_{i}-1} h(s) \frac{d s}{s}\right),
$$

where $\Omega$ is defined by (2.1). Therefore, the unique solution of fractional boundary value problem (2.2)-(2.3) is

$$
\begin{aligned}
u(t)= & \frac{(\log t)^{\alpha-1}}{\Omega} \int_{1}^{\infty} h(s) \frac{d s}{s}-\sum_{i=1}^{m} \frac{\lambda_{i}(\log t)^{\alpha-1}}{\Omega \Gamma\left(\alpha+\beta_{i}\right)} \int_{1}^{\eta}\left(\log \frac{\eta}{s}\right)^{\alpha+\beta_{i}-1} h(s) \frac{d s}{s} \\
& -\frac{1}{\Gamma(\alpha)} \int_{1}^{t}\left(\log \frac{t}{s}\right)^{\alpha-1} h(s) \frac{d s}{s} \\
= & \frac{(\log t)^{\alpha-1} \Gamma(\alpha)}{\Gamma(\alpha)-\sum_{i=1}^{m} \frac{\lambda_{i} \Gamma(\alpha)}{\Gamma\left(\alpha+\beta_{i}\right)}(\log \eta)^{\alpha+\beta_{i}-1}} \int_{1}^{\infty} \frac{h(s)}{\Gamma(\alpha)} \frac{d s}{s} \\
& -\sum_{i=1}^{m} \frac{\lambda_{i}(\log t)^{\alpha-1}}{\Omega \Gamma\left(\alpha+\beta_{i}\right)} \int_{1}^{\eta}\left(\log \frac{\eta}{s}\right)^{\alpha+\beta_{i}-1} h(s) \frac{d s}{s}-\frac{1}{\Gamma(\alpha)} \int_{1}^{t}\left(\log \frac{t}{s}\right)^{\alpha-1} h(s) \frac{d s}{s} \\
= & \frac{(\log t)^{\alpha-1}\left(\Gamma(\alpha)-\sum_{i=1}^{m} \frac{\lambda_{i} \Gamma(\alpha)}{\Gamma\left(\alpha+\beta_{i}\right)}(\log \eta)^{\alpha+\beta_{i}-1}+\sum_{i=1}^{m} \frac{\lambda_{i} \Gamma(\alpha)}{\Gamma\left(\alpha+\beta_{i}\right)}(\log \eta)^{\alpha+\beta_{i}-1}\right)}{\Gamma(\alpha)-\sum_{i=1}^{m} \frac{\lambda_{i} \Gamma(\alpha)}{\Gamma\left(\alpha+\beta_{i}\right)}(\log \eta)^{\alpha+\beta_{i}-1}} \\
& \times \int_{1}^{\infty} \frac{h(s)}{\Gamma(\alpha)} \frac{d s}{s}-\sum_{i=1}^{m} \frac{\lambda_{i}(\log t)^{\alpha-1}}{\Omega \Gamma\left(\alpha+\beta_{i}\right)} \int_{1}^{\eta}\left(\log \frac{\eta}{s}\right)^{\alpha+\beta_{i}-1} h(s) \frac{d s}{s} \\
& -\frac{1}{\Gamma(\alpha)} \int_{1}^{t}\left(\log \frac{t}{s}\right)^{\alpha-1} h(s) \frac{d s}{s} \\
= & (\log t)^{\alpha-1} \int_{1}^{\infty} \frac{h(s)}{\Gamma(\alpha)} \frac{d s}{s}+\sum_{i=1}^{m} \frac{\lambda_{i}(\log t)^{\alpha-1}}{\Omega \Gamma\left(\alpha+\beta_{i}\right)}(\log \eta)^{\alpha+\beta_{i}-1} \int_{1}^{\infty} h(s) \frac{d s}{s} \\
& -\sum_{i=1}^{m} \frac{\lambda_{i}(\log t)^{\alpha-1}}{\Omega \Gamma\left(\alpha+\beta_{i}\right)} \int_{1}^{\eta}\left(\log \frac{\eta}{s}\right)^{\alpha+\beta_{i}-1} h(s) \frac{d s}{s} \\
& -\frac{1}{\Gamma(\alpha)} \int_{1}^{t}\left(\log \frac{t}{s}\right)^{\alpha-1} h(s) \frac{d s}{s}
\end{aligned}
$$




$$
\begin{aligned}
= & \frac{1}{\Gamma(\alpha)} \int_{1}^{t}\left[(\log t)^{\alpha-1}-\left(\log \frac{t}{s}\right)^{\alpha-1}\right] h(s) \frac{d s}{s}+\frac{1}{\Gamma(\alpha)} \int_{t}^{\infty}(\log t)^{\alpha-1} h(s) \frac{d s}{s} \\
& +\sum_{i=1}^{m} \frac{\lambda_{i}(\log t)^{\alpha-1}}{\Omega \Gamma\left(\alpha+\beta_{i}\right)} \int_{1}^{\eta}\left[(\log \eta)^{\alpha+\beta_{i}-1}-\left(\log \frac{\eta}{s}\right)^{\alpha+\beta_{i}-1}\right] h(s) \frac{d s}{s} \\
& +\sum_{i=1}^{m} \frac{\lambda_{i}(\log t)^{\alpha-1}}{\Omega \Gamma\left(\alpha+\beta_{i}\right)} \int_{\eta}^{\infty}(\log \eta)^{\alpha+\beta_{i}-1} h(s) \frac{d s}{s} \\
= & \int_{1}^{\infty} g(t, s) h(s) \frac{d s}{s}+\sum_{i=1}^{m} \frac{\lambda_{i}(\log t)^{\alpha-1}}{\Omega \Gamma\left(\alpha+\beta_{i}\right)} \int_{1}^{\infty} g_{i}(\eta, s) h(s) \frac{d s}{s} \\
= & \int_{1}^{\infty} G(t, s) h(s) \frac{d s}{s} .
\end{aligned}
$$

The proof is completed.

Lemma 2.6 The Green's function $G(t, s)$ defined by (2.5) satisfies the following conditions:

$\left(C_{1}\right) G(t, s)$ is a continuous function for $(t, s) \in[1, \infty) \times[1, \infty)$;

$\left(C_{2}\right) G(t, s) \geq 0$ for all $s, t \in[1, \infty)$;

(C 3$) \frac{G(t, s)}{1+(\log t)^{\alpha-1}} \leq \frac{1}{\Gamma(\alpha)}+\sum_{i=1}^{m} \frac{\lambda_{i} g_{i}(\eta, s)}{\Omega \Gamma\left(\alpha+\beta_{i}\right)}$ for all $s, t \in[1, \infty)$;

$\left(\mathrm{C}_{4}\right) \min _{\eta \leq t \leq k \eta} \frac{G(t, s)}{1+(\log t)^{\alpha-1}} \geq \sum_{i=1}^{m} \frac{\lambda_{i}(\log \eta)^{\alpha-1} g_{i}(\eta, s)}{\Omega \Gamma\left(\alpha+\beta_{i}\right)\left(1+(\log \eta)^{\alpha-1}\right)}$ for $k>1$ and $s \in[1, \infty)$.

Proof It is easy to check that $\left(\mathrm{C}_{1}\right)$ and $\left(\mathrm{C}_{2}\right)$ hold.

To prove $\left(\mathrm{C}_{3}\right)$, we have, for $s, t \in[1, \infty)$,

$$
\begin{aligned}
\frac{G(t, s)}{1+(\log t)^{\alpha-1}} & =\frac{g(t, s)}{1+(\log t)^{\alpha-1}}+\sum_{i=1}^{m} \frac{\lambda_{i}(\log t)^{\alpha-1} g_{i}(\eta, s)}{\Omega \Gamma\left(\alpha+\beta_{i}\right)\left(1+(\log t)^{\alpha-1}\right)} \\
& \leq \frac{1}{\Gamma(\alpha)} \cdot \frac{(\log t)^{\alpha-1}}{1+(\log t)^{\alpha-1}}+\sum_{i=1}^{m} \frac{\lambda_{i}(\log t)^{\alpha-1} g_{i}(\eta, s)}{\Omega \Gamma\left(\alpha+\beta_{i}\right)\left(1+(\log t)^{\alpha-1}\right)} \\
& \leq \frac{1}{\Gamma(\alpha)}+\sum_{i=1}^{m} \frac{\lambda_{i} g_{i}(\eta, s)}{\Omega \Gamma\left(\alpha+\beta_{i}\right)}
\end{aligned}
$$

To prove $\left(\mathrm{C}_{4}\right)$, from $g(t, s) \geq 0$ and $g_{i}(\eta, s) \geq 0, i=1,2, \ldots, m$, for all $s, t \in[1, \infty)$, we have, for $k>1$,

$$
\begin{aligned}
\min _{\eta \leq t \leq k \eta} \frac{G(t, s)}{1+(\log t)^{\alpha-1}} & =\min _{\eta \leq t \leq k \eta}\left[\frac{g(t, s)}{1+(\log t)^{\alpha-1}}+\sum_{i=1}^{m} \frac{\lambda_{i}(\log t)^{\alpha-1} g_{i}(\eta, s)}{\Omega \Gamma\left(\alpha+\beta_{i}\right)\left(1+(\log t)^{\alpha-1}\right)}\right] \\
& \geq \min _{\eta \leq t \leq k \eta} \frac{g(t, s)}{1+(\log t)^{\alpha-1}}+\min _{\eta \leq t \leq k \eta} \sum_{i=1}^{m} \frac{\lambda_{i}(\log t)^{\alpha-1} g_{i}(\eta, s)}{\Omega \Gamma\left(\alpha+\beta_{i}\right)\left(1+(\log t)^{\alpha-1}\right)} \\
& \geq \min _{\eta \leq t \leq k \eta} \sum_{i=1}^{m} \frac{\lambda_{i}(\log t)^{\alpha-1} g_{i}(\eta, s)}{\Omega \Gamma\left(\alpha+\beta_{i}\right)\left(1+(\log t)^{\alpha-1}\right)} \\
& \geq \sum_{i=1}^{m} \frac{\lambda_{i}(\log \eta)^{\alpha-1} g_{i}(\eta, s)}{\Omega \Gamma\left(\alpha+\beta_{i}\right)\left(1+(\log \eta)^{\alpha-1}\right)}
\end{aligned}
$$

for $s \in[1, \infty)$. The proof is completed. 
In this paper, we will use the Banach space $E$, which is defined by

$$
E=\left\{u \in C([1, \infty), \mathbb{R}): \sup _{t \in[1, \infty)} \frac{|u(t)|}{1+(\log t)^{\alpha-1}}<\infty\right\}
$$

and is equipped with the norm

$$
\|u\|_{E}=\sup _{t \in[1, \infty)} \frac{|u(t)|}{1+(\log t)^{\alpha-1}} .
$$

Lemma $2.7\left(E,\|\cdot\|_{E}\right)$ is Banach space.

Proof Let $\left\{u_{n}\right\}_{n=1}^{\infty}$ be any Cauchy sequence in the space $\left(E,\|\cdot\|_{E}\right)$. Then $\forall \varepsilon>0, \exists N>0$ such that

$$
\left\|u_{n}-u_{m}\right\|_{E}=\sup _{t \in[1, \infty)} \frac{\left|u_{n}(t)-u_{m}(t)\right|}{1+(\log t)^{\alpha-1}}<\varepsilon
$$

for $n, m>N$. Therefore, any fixed $t_{0} \in[1, \infty)$, we have $\left\{u_{n}\left(t_{0}\right)\right\}_{n=1}^{\infty}$ is a Cauchy sequence in $\mathbb{R}$. In this way, we can associate to each $t \in[1, \infty)$ for a unique $u(t)$. Letting $n \rightarrow \infty$, we obtain $\left|u(t)-u_{m}(t)\right| \leq \varepsilon$ for all $m>N$ and $t \in[1, \infty)$. It is easy to show that $u_{m} \rightarrow u$ in $E$ as $m \rightarrow \infty$. Therefore, we see that $\left(E,\|\cdot\|_{E}\right)$ is Banach space.

Lemma 2.8 Let $U \subset E$ be a bounded set. Then $U$ is relatively compact in $E$ if the following conditions hold:

(i) for any $u(t) \in U, \frac{u(t)}{1+(\log t)^{\alpha-1}}$ is equicontinuous on any compact interval of $[1, \infty)$;

(ii) for any $\varepsilon>0$, there exists a constant $T=T(\varepsilon)>0$ such that

$$
\left|\frac{u\left(t_{1}\right)}{1+\left(\log t_{1}\right)^{\alpha-1}}-\frac{u\left(t_{2}\right)}{1+\left(\log t_{2}\right)^{\alpha-1}}\right|<\varepsilon
$$

for any $t_{1}, t_{2} \geq T$ and $u \in U$.

Proof Evidently, it is sufficient to prove that $U$ is totally bounded. In the following we divide the proof into two steps.

Step 1 . Let us consider the case $t \in[1, T]$.

Define

$$
U_{[1, T]}=\{u(t): u(t) \in U, t \in[1, T]\} .
$$

Then clearly, $U_{[1, T]}$, with the norm $\|u\|_{\infty}=\sup _{t \in[1, T]} \frac{|u(t)|}{1+(\log t)^{\alpha-1}}$ is a Banach space. The condition (i) combined with the Arzelá-Ascoli theorem indicates that $U_{[1, T]}$ is relatively compact, hence $U_{[1, T]}$ is totally bounded, namely, for any $\varepsilon>0$, there exist finitely many balls $B_{\varepsilon}\left(u_{i}\right)$ such that

$$
U_{[1, T]} \subset \bigcup_{i=1}^{n} B_{\varepsilon}\left(u_{i}\right),
$$


where

$$
B_{\varepsilon}\left(u_{i}\right)=\left\{u(t) \in U_{[1, T]}:\left\|u-u_{i}\right\|_{\infty}=\sup _{t \in[1, T]}\left|\frac{u(t)}{1+(\log t)^{\alpha-1}}-\frac{u_{i}(t)}{1+(\log t)^{\alpha-1}}\right|<\varepsilon\right\} .
$$

Step 2. Define

$$
U_{i}=\left\{u(t) \in U: u_{[1, T]} \in B_{\varepsilon}\left(u_{i}\right)\right\} .
$$

It is clear that $U_{[1, T]} \subset \bigcup_{1 \leq i \leq n} U_{i[1, T]}$. Now let us take $u_{i} \in U_{i}$; then $U$ can be covered by the balls $B_{3 \varepsilon}\left(u_{i}\right), i=1,2, \ldots, n$ where

$$
B_{3 \varepsilon}\left(u_{i}\right)=\left\{u(t) \in U:\left\|u-u_{i}\right\|_{E}<3 \varepsilon\right\} .
$$

In fact, for $u(t) \in U$, the arguments in Step 1 imply that there exist $i$ such that $u_{[1, T]} \in B_{\varepsilon}\left(u_{i}\right)$. Hence, for $t \in[1, T]$, we have

$$
\left|\frac{u(t)}{1+(\log t)^{\alpha-1}}-\frac{u_{i}(t)}{1+(\log t)^{\alpha-1}}\right|<\varepsilon .
$$

For $t \in[T,+\infty),(2.11)$, and (2.12) yield

$$
\begin{aligned}
& \left|\frac{u(t)}{1+(\log t)^{\alpha-1}}-\frac{u_{i}(t)}{1+(\log t)^{\alpha-1}}\right| \\
& \quad \leq\left|\frac{u(t)}{1+(\log t)^{\alpha-1}}-\frac{u(T)}{1+(\log T)^{\alpha-1}}\right|+\left|\frac{u(T)}{1+(\log T)^{\alpha-1}}-\frac{u_{i}(T)}{1+(\log T)^{\alpha-1}}\right| \\
& \quad+\left|\frac{u_{i}(T)}{1+(\log T)^{\alpha-1}}-\frac{u_{i}(t)}{1+(\log t)^{\alpha-1}}\right| \\
& \quad<\varepsilon+\varepsilon+\varepsilon=3 \varepsilon .
\end{aligned}
$$

Equations (2.12) and (2.13) show that $\left\|u(t)-u_{i}(t)\right\|_{E}<3 \varepsilon$. Therefore, $U$ is totally bounded and Lemma 2.8 is proved.

We define the cone $P \subset E$ by

$$
P=\{u \in E: u(t) \geq 0 \text { on }[1, \infty)\}
$$

and the operator $T: P \rightarrow E$ by

$$
T u(t)=\int_{1}^{\infty} G(t, s) a(s) f(u(s)) \frac{d s}{s}, \quad t \in[1, \infty),
$$

where $G(t, s)$ defined by (2.5).

Throughout this paper, we assume that the following conditions hold:

$\left(\mathrm{A}_{1}\right) f \in C([0, \infty),[0, \infty)), f(u) \neq 0$ on any subinterval of $(0, \infty)$ and $f\left(\left(1+(\log t)^{\alpha-1}\right) u\right)$ is bounded on $[0, \infty)$; 
$\left(\mathrm{A}_{2}\right) a:[1, \infty) \rightarrow[0, \infty)$ is not identical zero on any closed subinterval of $[1, \infty)$ and

$$
0<\int_{1}^{\infty} a(s) \frac{d s}{s}<\infty
$$

Lemma 2.9 Let $\left(\mathrm{A}_{1}\right)$ and $\left(\mathrm{A}_{2}\right)$ hold. Then $T: P \rightarrow P$ is completely continuous.

Proof We divide the proof into four steps.

Step 1: We show that $T$ is uniformly bounded on $P$.

From the definition of $E$, we can choose $r_{0}$ such that $\sup _{n \in \mathbb{N}}\left\|u_{n}\right\|_{E}<r_{0}$. Let $B_{r_{0}}=$ $\sup \left\{f\left(\left(1+(\log t)^{\alpha-1}\right) u\right), u \in\left[1, r_{0}\right]\right\}$ and $\Phi$ be any bounded subset of $P$, then there exists $r>0$ such that $\|u\|_{E} \leq r$ for all $u \in \Phi$. From $\left(\mathrm{C}_{3}\right)$, it follows that

$$
\begin{aligned}
\|T u\|_{E} & =\sup _{t \in[1, \infty)} \frac{1}{1+(\log t)^{\alpha-1}}\left|\int_{1}^{\infty} G(t, s) a(s) f(u(s)) \frac{d s}{s}\right| \\
& \leq \int_{1}^{\infty}\left(\frac{1}{\Gamma(\alpha)}+\sum_{i=1}^{m} \frac{\lambda_{i} g_{i}(\eta, s)}{\Omega \Gamma\left(\alpha+\beta_{i}\right)}\right) a(s) f(u(s)) \frac{d s}{s} \\
& \leq\left(\frac{1}{\Gamma(\alpha)}+\sum_{i=1}^{m} \frac{\lambda_{i}(\log \eta)^{\alpha+\beta_{i}-1}}{\Omega \Gamma\left(\alpha+\beta_{i}\right)}\right) B_{r} \int_{1}^{\infty} a(s) \frac{d s}{s}<\infty
\end{aligned}
$$

for $u \in \Phi$. Therefore $T \Phi$ is uniformly bounded.

Step 2: We show that $T$ is equicontinuous on any compact interval of $[1, \infty)$.

For any $S>1, t_{1}, t_{2} \in[1, S]$, and $u \in \Phi$, without loss of generality, we assume that $t_{1}<t_{2}$. In fact,

$$
\begin{aligned}
& \left|\frac{T u\left(t_{2}\right)}{1+\left(\log t_{2}\right)^{\alpha-1}}-\frac{T u\left(t_{1}\right)}{1+\left(\log t_{1}\right)^{\alpha-1}}\right| \\
& =\left|\int_{1}^{\infty} \frac{G\left(t_{2}, s\right)}{1+\left(\log t_{2}\right)^{\alpha-1}} a(s) f(u(s)) \frac{d s}{s}-\int_{1}^{\infty} \frac{G\left(t_{1}, s\right)}{1+\left(\log t_{1}\right)^{\alpha-1}} a(s) f(u(s)) \frac{d s}{s}\right| \\
& \leq\left|\int_{1}^{\infty}\left(\frac{g\left(t_{2}, s\right)}{1+\left(\log t_{2}\right)^{\alpha-1}}-\frac{g\left(t_{1}, s\right)}{1+\left(\log t_{1}\right)^{\alpha-1}}\right) a(s) f(u(s)) \frac{d s}{s}\right| \\
& +\left|\int_{1}^{\infty}\left(\frac{\left(\log t_{2}\right)^{\alpha-1}}{1+\left(\log t_{2}\right)^{\alpha-1}}-\frac{\left(\log t_{1}\right)^{\alpha-1}}{1+\left(\log t_{1}\right)^{\alpha-1}}\right) \sum_{i=1}^{m} \frac{\lambda_{i} g_{i}(\eta, s)}{\Omega \Gamma\left(\alpha+\beta_{i}\right)} a(s) f(u(s)) \frac{d s}{s}\right| \\
& \leq \int_{1}^{\infty}\left|\frac{g\left(t_{2}, s\right)}{1+\left(\log t_{2}\right)^{\alpha-1}}-\frac{g\left(t_{1}, s\right)}{1+\left(\log t_{2}\right)^{\alpha-1}}\right| a(s) f(u(s)) \frac{d s}{s} \\
& +\int_{1}^{\infty}\left|\frac{g\left(t_{1}, s\right)}{1+\left(\log t_{2}\right)^{\alpha-1}}-\frac{g\left(t_{1}, s\right)}{1+\left(\log t_{1}\right)^{\alpha-1}}\right| a(s) f(u(s)) \frac{d s}{s} \\
& +\int_{1}^{\infty}\left|\frac{\left(\log t_{2}\right)^{\alpha-1}}{1+\left(\log t_{2}\right)^{\alpha-1}}-\frac{\left(\log t_{1}\right)^{\alpha-1}}{1+\left(\log t_{1}\right)^{\alpha-1}}\right| \sum_{i=1}^{m} \frac{\lambda_{i} g_{i}(\eta, s)}{\Omega \Gamma\left(\alpha+\beta_{i}\right)} a(s) f(u(s)) \frac{d s}{s} \\
& =\int_{1}^{\infty}\left|\frac{g\left(t_{2}, s\right)}{1+\left(\log t_{2}\right)^{\alpha-1}}-\frac{g\left(t_{1}, s\right)}{1+\left(\log t_{2}\right)^{\alpha-1}}\right| a(s) f(u(s)) \frac{d s}{s} \\
& +\int_{1}^{\infty} \frac{\left(\log t_{2}\right)^{\alpha-1}-\left(\log t_{1}\right)^{\alpha-1}}{\left(1+\left(\log t_{2}\right)^{\alpha-1}\right)\left(1+\left(\log t_{1}\right)^{\alpha-1}\right)} g\left(t_{1}, s\right) a(s) f(u(s)) \frac{d s}{s} \\
& +\int_{1}^{\infty} \frac{\left(\log t_{2}\right)^{\alpha-1}-\left(\log t_{1}\right)^{\alpha-1}}{\left(1+\left(\log t_{2}\right)^{\alpha-1}\right)\left(1+\left(\log t_{1}\right)^{\alpha-1}\right)} \sum_{i=1}^{m} \frac{\lambda_{i} g_{i}(\eta, s)}{\Omega \Gamma\left(\alpha+\beta_{i}\right)} a(s) f(u(s)) \frac{d s}{s} .
\end{aligned}
$$


We now consider

$$
\begin{aligned}
\int_{1}^{\infty} \mid & \frac{g\left(t_{2}, s\right)}{1+\left(\log t_{2}\right)^{\alpha-1}}-\frac{g\left(t_{1}, s\right)}{1+\left(\log t_{2}\right)^{\alpha-1}} \mid a(s) f(u(s)) \frac{d s}{s} \\
= & \int_{1}^{t_{1}}\left|\frac{g\left(t_{2}, s\right)}{1+\left(\log t_{2}\right)^{\alpha-1}}-\frac{g\left(t_{1}, s\right)}{1+\left(\log t_{2}\right)^{\alpha-1}}\right| a(s) f(u(s)) \frac{d s}{s} \\
& +\int_{t_{1}}^{t_{2}}\left|\frac{g\left(t_{2}, s\right)}{1+\left(\log t_{2}\right)^{\alpha-1}}-\frac{g\left(t_{1}, s\right)}{1+\left(\log t_{2}\right)^{\alpha-1}}\right| a(s) f(u(s)) \frac{d s}{s} \\
& +\int_{t_{2}}^{\infty}\left|\frac{g\left(t_{2}, s\right)}{1+\left(\log t_{2}\right)^{\alpha-1}}-\frac{g\left(t_{1}, s\right)}{1+\left(\log t_{2}\right)^{\alpha-1}}\right| a(s) f(u(s)) \frac{d s}{s} \\
\leq & \frac{1}{\Gamma(\alpha)} \int_{1}^{t_{1}} \frac{\left(\log t_{2}\right)^{\alpha-1}-\left(\log t_{1}\right)^{\alpha-1}+\left(\log \frac{t_{2}}{s}\right)^{\alpha-1}-\left(\log \frac{t_{1}}{s}\right)^{\alpha-1}}{1+\left(\log t_{2}\right)^{\alpha-1}} a(s) f(u(s)) \frac{d s}{s} \\
& +\frac{1}{\Gamma(\alpha)} \int_{t_{1}}^{t_{2}} \frac{\left(\log t_{2}\right)^{\alpha-1}-\left(\log t_{1}\right)^{\alpha-1}+\left(\log \frac{t_{2}}{s}\right)^{\alpha-1}}{1+\left(\log t_{2}\right)^{\alpha-1}} a(s) f(u(s)) \frac{d s}{s} \\
& +\frac{1}{\Gamma(\alpha)} \int_{t_{2}}^{\infty} \frac{\left(\log t_{2}\right)^{\alpha-1}-\left(\log t_{1}\right)^{\alpha-1}}{1+\left(\log t_{2}\right)^{\alpha-1}} a(s) f(u(s)) \frac{d s}{s} \\
\rightarrow & 0 \quad \text { uniformly as } t_{1} \rightarrow t_{2} .
\end{aligned}
$$

Similarly, we have

$$
\int_{1}^{\infty} \frac{\left(\log t_{2}\right)^{\alpha-1}-\left(\log t_{1}\right)^{\alpha-1}}{\left(1+\left(\log t_{2}\right)^{\alpha-1}\right)\left(1+\left(\log t_{1}\right)^{\alpha-1}\right)} g\left(t_{1}, s\right) a(s) f(u(s)) \frac{d s}{s} \rightarrow 0
$$

uniformly as $t_{1} \rightarrow t_{2}$, and

$$
\int_{1}^{\infty} \frac{\left(\log t_{2}\right)^{\alpha-1}-\left(\log t_{1}\right)^{\alpha-1}}{\left(1+\left(\log t_{2}\right)^{\alpha-1}\right)\left(1+\left(\log t_{1}\right)^{\alpha-1}\right)} \sum_{i=1}^{m} \frac{\lambda_{i} g_{i}(\eta, s)}{\Omega \Gamma\left(\alpha+\beta_{i}\right)} a(s) f(u(s)) \frac{d s}{s} \rightarrow 0
$$

uniformly as $t_{1} \rightarrow t_{2}$.

Hence, from (2.15), (2.16), and (2.17), we get

$$
\left|\frac{T u\left(t_{2}\right)}{1+\left(\log t_{2}\right)^{\alpha-1}}-\frac{T u\left(t_{1}\right)}{1+\left(\log t_{1}\right)^{\alpha-1}}\right| \rightarrow 0 \quad \text { uniformly as } t_{1} \rightarrow t_{2} .
$$

Thus $T \Phi$ is equicontinuous on $[1, \infty)$.

Step 3: We show that $T$ is equiconvergent at $\infty$.

For any $u \in \Phi$, we have

$$
\int_{1}^{\infty} a(s) f(u(s)) \frac{d s}{s} \leq B_{r} \int_{1}^{\infty} a(s) \frac{d s}{s}<\infty
$$

and

$$
\begin{aligned}
& \lim _{t \rightarrow \infty}\left|\frac{(T u)(t)}{1+(\log t)^{\alpha-1}}\right| \\
& \quad=\lim _{t \rightarrow \infty}\left|\frac{1}{1+(\log t)^{\alpha-1}} \int_{1}^{\infty} G(t, s) a(s) f(u(s)) \frac{d s}{s}\right|
\end{aligned}
$$




$$
\begin{aligned}
& \leq \lim _{t \rightarrow \infty} \int_{1}^{\infty}\left(\frac{1}{\Gamma(\alpha)} \cdot \frac{(\log t)^{\alpha-1}}{1+(\log t)^{\alpha-1}}+\frac{(\log t)^{\alpha-1}}{1+(\log t)^{\alpha-1}} \cdot \sum_{i=1}^{m} \frac{\lambda_{i} g_{i}(\eta, s)}{\Omega \Gamma\left(\alpha+\beta_{i}\right)}\right) a(s) f(u(s)) \frac{d s}{s} \\
& \leq \int_{1}^{\infty}\left(\frac{1}{\Gamma(\alpha)}+\sum_{i=1}^{m} \frac{\lambda_{i} g_{i}(\eta, s)}{\Omega \Gamma\left(\alpha+\beta_{i}\right)}\right) a(s) f(u(s)) \frac{d s}{s} \\
& \leq\left(\frac{1}{\Gamma(\alpha)}+\sum_{i=1}^{m} \frac{\lambda_{i}(\log \eta)^{\alpha+\beta_{i}-1}}{\Omega \Gamma\left(\alpha+\beta_{i}\right)}\right) B_{r} \int_{1}^{\infty} a(s) \frac{d s}{s} \\
& <\infty
\end{aligned}
$$

Hence, $T \Phi$ is equiconvergent at infinity.

Step 4: We show that $T$ is continuous.

Let $u_{n} \rightarrow u$ as $\rightarrow \infty$ in $P$. We have

$$
\int_{1}^{\infty} a(s) f(u(s)) \frac{d s}{s}<\infty .
$$

Hence the Lebesgue dominated convergence and the continuity of $f$ guarantee that

$$
\int_{1}^{\infty} a(s) f\left(u_{n}(s)\right) \frac{d s}{s} \rightarrow \int_{1}^{\infty} a(s) f(u(s)) \frac{d s}{s} \quad \text { as } n \rightarrow \infty .
$$

Therefore, we get

$$
\begin{aligned}
& \left\|T u_{n}-T u\right\|_{E} \\
& \quad=\sup _{t \in[1, \infty)} \frac{1}{1+(\log t)^{\alpha-1}}\left|T u_{n}-T u\right| \\
& \quad=\sup _{t \in[1, \infty)}\left|\int_{1}^{\infty} \frac{G(t, s)}{1+(\log t)^{\alpha-1}} a(s)\left[f\left(u_{n}(s)\right)-f(u(s))\right] \frac{d s}{s}\right| \\
& \leq\left(\frac{1}{\Gamma(\alpha)}+\sum_{i=1}^{m} \frac{\lambda_{i}(\log \eta)^{\alpha+\beta_{i}-1}}{\Omega \Gamma\left(\alpha+\beta_{i}\right)}\right)\left|\int_{1}^{\infty} a(s) f\left(u_{n}(s)\right) \frac{d s}{s}-\int_{1}^{\infty} a(s) f(u(s)) \frac{d s}{s}\right| \\
& \rightarrow 0 \text { as } n \rightarrow \infty .
\end{aligned}
$$

So, $T$ is continuous.

Using Lemma 2.8, we see that $T: P \rightarrow P$ is completely continuous. The proof is completed.

\section{Existence of at least three positive solutions}

In this section we use the Leggett-Williams fixed point theorem to prove the existence of at least three positive solutions.

For convenience, we denote

$$
\begin{aligned}
& M=\left(\frac{1}{\Gamma(\alpha)}+\sum_{i=1}^{m} \frac{\lambda_{i}(\log \eta)^{\alpha+\beta_{i}-1}}{\Omega \Gamma\left(\alpha+\beta_{i}\right)}\right) \int_{1}^{\infty} a(s) \frac{d s}{s}>0, \\
& m=\sum_{i=1}^{m} \frac{\lambda_{i}(\log \eta)^{2 \alpha+\beta_{i}-2}}{\Omega \Gamma\left(\alpha+\beta_{i}\right)\left(1+(\log \eta)^{\alpha-1}\right)} \int_{\eta}^{k \eta} a(s) \frac{d s}{s}>0 .
\end{aligned}
$$


Definition 3.1 A continuous mapping $\theta: P \rightarrow[1, \infty)$ is said to be a nonnegative continuous concave functional on the cone $P$ of a real Banach space $E$ provided that

$$
\theta(\lambda u+(1-\lambda) v) \geq \lambda \theta(u)+(1-\lambda) \theta(v)
$$

for all $u, v \in P$ and $\lambda \in[0,1]$.

Let $a, b, d>0$ be constants. We defined $P_{d}=\{u \in P:\|u\|<d\}, \bar{P}_{d}=\{u \in P:\|u\| \leq d\}$, and $P(\theta, a, b)=\{u \in P: \theta(u) \geq a,\|u\| \leq b\}$.

Theorem 3.2 [27] Let $P$ be a cone in the real Banach space $E$ and $c>0$ be a constant. Assume that there exists a concave nonnegative continuous functional $\theta$ on $P$ with $\theta(u) \leq$ $\|u\|$ for all $u \in \bar{P}_{c}$. Let $T: \bar{P}_{c} \rightarrow \bar{P}_{c}$ be a completely continuous operator. Suppose that there exist constants $0<a<b<d \leq c$ such that the following conditions hold:

(i) $\{u \in P(\theta, b, d): \theta(u)>b\} \neq \emptyset$ and $\theta(T u)>b$ for $u \in P(\theta, b, d)$;

(ii) $\|T u\|<a$ for $u \leq a$;

(iii) $\theta(T u)>b$ for $u \in P(\theta, b, c)$ with $\|T u\|>d$.

Then $T$ has at least three fixed points $u_{1}, u_{2}$, and $u_{3}$ in $\bar{P}_{c}$. Furthermore, $\left\|u_{1}\right\|<a, b<\theta\left(u_{2}\right)$, $a<\left\|u_{3}\right\|$ with $\theta\left(u_{3}\right)<b$.

Theorem 3.3 Suppose conditions $\left(\mathrm{A}_{1}\right)$ and $\left(\mathrm{A}_{2}\right)$ hold. Let $0<a<b<d \leq c$ and suppose that $f$ satisfies the following conditions:

$\left(\mathrm{A}_{3}\right) f\left(\left(1+(\log t)^{\alpha-1}\right) u\right)<\frac{c}{M}$ for all $(t, u) \in[1, \infty) \times[0, c]$;

( $\left.\mathrm{A}_{4}\right) f\left(\left(1+(\log t)^{\alpha-1}\right) u\right)>\frac{b}{m}$ for all $(t, u) \in[\eta, k \eta] \times[b, c]$;

( $\left.\mathrm{A}_{5}\right) f\left(\left(1+(\log t)^{\alpha-1}\right) u\right)<\frac{a}{M}$ for all $(t, u) \in[1, \infty) \times[0, a]$.

Then fractional boundary value problem (1.9)-(1.10) has at least three positive solutions $u_{1}$, $u_{2}$, and $u_{3}$ satisfying

$$
\left\|u_{1}\right\|_{E}<a, \quad b<\theta\left(u_{2}\right)
$$

and

$$
a<\left\|u_{3}\right\|_{E} \quad \text { with } \theta\left(u_{3}\right)<b .
$$

Proof We will show that the conditions of the Leggett-Williams fixed point theorem are satisfied for the operator $T$ defined by (2.14). We defined a nonnegative functional on $E$ by

$$
\theta(u)=\min _{\eta \leq t \leq k \eta} \frac{u(t)}{1+(\log t)^{\alpha-1}} .
$$

For $u \in \bar{P}_{c}$, we have $\|u\|_{E} \leq c$, that is,

$$
0 \leq \frac{u(t)}{1+(\log t)^{\alpha-1}} \leq c \text { for } t \in[1, \infty)
$$


Then assumption $\left(\mathrm{A}_{3}\right)$ implies

$$
f(u)<\frac{c}{M} \quad \text { for }(t, u) \in[1, \infty) \times[0, c]
$$

Therefore,

$$
\begin{aligned}
\|T u\|_{E} & =\sup _{t \in[1, \infty)} \frac{1}{1+(\log t)^{\alpha-1}}\left|\int_{1}^{\infty} G(t, s) a(s) f(u(s)) \frac{d s}{s}\right| \\
& <\left(\frac{1}{\Gamma(\alpha)}+\sum_{i=1}^{m} \frac{\lambda_{i}(\log \eta)^{\alpha+\beta_{i}-1}}{\Omega \Gamma\left(\alpha+\beta_{i}\right)}\right) \frac{c}{M} \int_{1}^{\infty} a(s) \frac{d s}{s}=c .
\end{aligned}
$$

Hence, $T: P_{c} \rightarrow P_{c}$ and by Lemma $2.9, T$ is completely continuous. It follows from condition $\left(\mathrm{A}_{5}\right)$, using the above argument, that if $u \in \bar{P}_{a}$ then $\|T u\|_{E}<a$. Therefore, condition (ii) of Theorem 3.2 holds.

Let

$$
u^{*}(t)=\frac{b+c}{2}\left(1+(\log t)^{\alpha-1}\right), \quad t \in[1, \infty)
$$

It is obvious that $u^{*}(t) \in P$ and $\left\|u^{*}\right\|=\frac{b+c}{2}<c$. From the definition of $\theta(u)$, then

$$
\theta\left(u^{*}\right)=\frac{b+c}{2}>b
$$

Hence, we get

$$
u^{*} \in\{x \in P(\theta, b, d): \theta(x)>b\} \neq \emptyset .
$$

Moreover, for $u \in P(\theta, b, d)$, it follows that

$$
b \leq \frac{u(t)}{1+(\log t)^{\alpha-1}} \leq c \quad \text { for } t \in[\eta, k \eta] .
$$

Then assumption $\left(\mathrm{A}_{4}\right)$ implies

$$
f(u)>\frac{b}{m} \quad \text { for }(t, u) \in[\eta, k \eta] \times[b, c] .
$$

So, we have

$$
\begin{aligned}
\theta(T u) & =\min _{\eta \leq t \leq k \eta} \frac{T u(t)}{1+(\log t)^{\alpha-1}} \\
& \geq \int_{1}^{\infty} \min _{\eta \leq t \leq k \eta} \frac{G(t, s)}{1+(\log t)^{\alpha-1}} a(s) f(u(s)) \frac{d s}{s} \\
& \geq \sum_{i=1}^{m} \frac{\lambda_{i}(\log \eta)^{\alpha-1}}{\Omega \Gamma\left(\alpha+\beta_{i}\right)\left(1+(\log \eta)^{\alpha-1}\right)} \int_{1}^{\infty} g_{i}(\eta, s) a(s) f(u(s)) \frac{d s}{s} \\
& \geq \sum_{i=1}^{m} \frac{\lambda_{i}(\log \eta)^{\alpha-1}}{\Omega \Gamma\left(\alpha+\beta_{i}\right)\left(1+(\log \eta)^{\alpha-1}\right)} \int_{\eta}^{k \eta} g_{i}(\eta, s) a(s) f(u(s)) \frac{d s}{s}
\end{aligned}
$$




$$
\begin{aligned}
& >\sum_{i=1}^{m} \frac{\lambda_{i}(\log \eta)^{2 \alpha+\beta_{i}-2}}{\Omega \Gamma\left(\alpha+\beta_{i}\right)\left(1+(\log \eta)^{\alpha-1}\right)} \frac{b}{m} \int_{\eta}^{k \eta} a(s) \frac{d s}{s} \\
& =b .
\end{aligned}
$$

Thus $\theta(T u)>b$ for all $u \in P(\theta, b, d)$. This shows that condition (i) of Theorem 3.2 holds.

Finally, we assume that $u \in P(\theta, b, c)$ with $\|T u\|_{E}>d$; then $\|u\|_{E} \leq c$ and $b \leq \frac{u(t)}{1+(\log t)^{\alpha-1}} \leq c$ and from assumption $\left(\mathrm{A}_{4}\right)$ we can show $\theta(\mathrm{Tu})>b$. So, condition (iii) of Theorem 3.2 is satisfied. As a consequence of Theorem 3.2 implies that boundary value problem (1.9)(1.10) has at least three positive solutions $u_{1}, u_{2}$, and $u_{3}$ such that

$$
\left\|u_{1}\right\|_{E}<a, \quad b<\theta\left(u_{2}\right) \quad \text { and } \quad a<\left\|u_{3}\right\|_{E} \quad \text { with } \theta\left(u_{3}\right)<b
$$

The proof is completed.

\section{Existence of at least one positive solution}

In this section we use the Guo-Krasnoselskii fixed point theorem to prove the existence of at least one positive solution.

Theorem 4.1 [28] Let E be a Banach space, and let $P \subset E$ be a cone. Assume that $\Omega_{1}, \Omega_{2}$ are open subsets of $E$ with $0 \in \Omega_{1}, \bar{\Omega}_{1} \subset \Omega_{2}$, and let $T: P \cap\left(\bar{\Omega}_{2} \backslash \Omega_{1}\right) \rightarrow P$ be a completely continuous operator such that:

(i) $\|T u\| \geq\|u\|, u \in P \cap \partial \Omega_{1}$, and $\|T u\| \leq\|u\|, u \in P \cap \partial \Omega_{2}$; or

(ii) $\|T u\| \leq\|u\|, u \in P \cap \partial \Omega_{1}$, and $\|T u\| \geq\|u\|, u \in P \cap \partial \Omega_{2}$.

Then $T$ has a fixed point in $P \cap\left(\bar{\Omega}_{2} \backslash \Omega_{1}\right)$.

Theorem 4.2 Suppose conditions $\left(\mathrm{A}_{1}\right)$ and $\left(\mathrm{A}_{2}\right)$ hold. Let $r_{2}>r_{1}>0, \rho_{1} \in\left(m^{-1}, \infty\right), \rho_{2} \in$ $\left(0, M^{-1}\right)$, and suppose that $f$ satisfies the following conditions:

(A $\left.\mathrm{A}_{6}\right) f\left(\left(1+(\log t)^{\alpha-1}\right) u\right) \geq \rho_{1} r_{1}$ for all $(t, u) \in[1, \infty) \times\left[0, r_{1}\right]$;

$\left(\mathrm{A}_{7}\right) f\left(\left(1+(\log t)^{\alpha-1}\right) u\right) \leq \rho_{2} r_{2}$ for all $(t, u) \in[1, \infty) \times\left[0, r_{2}\right]$.

Then fractional boundary value problem (1.9)-(1.10) has at least one positive solution u such that

$$
r_{1}<\|u\|_{E}<r_{2}
$$

Proof We will show that the condition (i) of Theorem 4.1 is satisfied. By Lemma 2.9, the operator $T: P \rightarrow P$ is completely continuous.

Let $\Phi_{1}=\left\{u \in E:\|u\|_{E}<r_{1}\right\}$, then for any $u \in P \cap \partial \Phi_{1}$, we have

$$
0 \leq \frac{u(t)}{1+(\log t)^{\alpha-1}} \leq r_{1} \quad \text { for all } t \in[1, \infty)
$$

Then assumption $\left(\mathrm{A}_{6}\right)$ implies

$$
f(u) \geq \rho_{1} r_{1} \quad \text { for }(t, u) \in[1, \infty) \times\left[0, r_{1}\right]
$$


Therefore, for $t \in[1, \infty)$, we get

$$
\begin{aligned}
\|T u\|_{E} & =\sup _{t \in[1, \infty)} \frac{1}{1+(\log t)^{\alpha-1}}\left|\int_{1}^{\infty} G(t, s) a(s) f(u(s)) \frac{d s}{s}\right| \\
& \geq \min _{t \in[\eta, k \eta]} \int_{1}^{\infty} \frac{G(t, s)}{1+(\log t)^{\alpha-1}} a(s) f(u(s)) \frac{d s}{s} \\
& \geq \int_{1}^{\infty} \min _{t \in[\eta, k \eta]} \frac{G(t, s)}{1+(\log t)^{\alpha-1}} a(s) f(u(s)) \frac{d s}{s} \\
& \geq \sum_{i=1}^{m} \frac{\lambda_{i}(\log \eta)^{2 \alpha+\beta_{i}-2}}{\Omega \Gamma\left(\alpha+\beta_{i}\right)\left(1+(\log \eta)^{\alpha-1}\right)} \rho_{1} r_{1} \int_{\eta}^{k \eta} a(s) \frac{d s}{s} \\
& \geq r_{1}=\|u\|_{E} \quad \text { for } u \in P \cap \partial \Phi_{1} .
\end{aligned}
$$

Let $\Phi_{2}=\left\{u \in E:\|u\|_{E}<r_{2}\right\}$, then for any $u \in P \cap \partial \Phi_{2}$, it follows that

$$
0 \leq \frac{u(t)}{1+(\log t)^{\alpha-1}} \leq r_{2} \quad \text { for all } t \in[1, \infty)
$$

For $t \in[1, \infty)$, assumption $\left(\mathrm{A}_{7}\right)$ yields

$$
\begin{aligned}
\|T u\|_{E} & =\sup _{t \in[1, \infty)} \frac{1}{1+(\log t)^{\alpha-1}}\left|\int_{1}^{\infty} G(t, s) a(s) f(u(s)) \frac{d s}{s}\right| \\
& \leq \int_{1}^{\infty}\left(\frac{1}{\Gamma(\alpha)}+\sum_{i=1}^{m} \frac{\lambda_{i} g_{i}(\eta, s)}{\Omega \Gamma\left(\alpha+\beta_{i}\right)}\right) a(s) f(u(s)) \frac{d s}{s} \\
& \leq\left(\frac{1}{\Gamma(\alpha)}+\sum_{i=1}^{m} \frac{\lambda_{i}(\log \eta)^{\alpha+\beta_{i}-1}}{\Omega \Gamma\left(\alpha+\beta_{i}\right)}\right) \rho_{2} r_{2} \int_{1}^{\infty} a(s) \frac{d s}{s} \\
& \leq r_{2}=\|u\|_{E} \quad \text { for } u \in P \cap \partial \Phi_{2} .
\end{aligned}
$$

Hence, from (4.1), (4.2), and condition (i) of Theorem 4.1, it follows that $T$ has a fixed point in $P \cap\left(\bar{\Phi}_{2} \backslash \Phi_{1}\right)$. Therefore, the boundary value problem (1.9)-(1.10) has at least one positive solution such that

$$
r_{1}<\|u\|_{E}<r_{2} .
$$

The proof is completed.

Similarly to the previous theorem we can show the following theorem.

Theorem 4.3 Suppose conditions $\left(\mathrm{A}_{1}\right)$ and $\left(\mathrm{A}_{2}\right)$ hold. Let $r_{2}>r_{1}>0, \rho_{1} \in\left(m^{-1}, \infty\right), \rho_{2} \in$ $\left(0, M^{-1}\right)$, and suppose that $f$ satisfies the following conditions:

( $\left.\mathrm{A}_{8}\right) f\left(\left(1+(\log t)^{\alpha-1}\right) u\right) \leq \rho_{2} r_{1}$ for all $(t, u) \in[1, \infty) \times\left[0, r_{1}\right]$;

( $\left.\mathrm{A}_{9}\right) f\left(\left(1+(\log t)^{\alpha-1}\right) u\right) \geq \rho_{1} r_{2}$ for all $(t, u) \in[1, \infty) \times\left[0, r_{2}\right]$.

Then fractional boundary value problem (1.9)-(1.10) has at least one positive solution $u$ such that

$$
r_{1}<\|u\|_{E}<r_{2} .
$$




\section{Examples}

Example 5.1 We consider the following Hadamard fractional differential equation with nonlocal boundary conditions on an unbounded domain:

$$
\left\{\begin{array}{l}
D^{\frac{3}{2}} u(t)+e^{-t} f(u(t))=0, \quad t \in(1, \infty) \\
u(1)=0, \quad D^{\frac{1}{2}} u(\infty)=\frac{1}{10} I^{\frac{3}{4}} u\left(\frac{5}{2}\right)+\pi I^{\frac{5}{2}} u\left(\frac{5}{2}\right)+\frac{1}{3} I^{\frac{7}{2}} u\left(\frac{5}{2}\right)
\end{array}\right.
$$

where

$$
f(u(t))= \begin{cases}\cos ^{2}\left(\left(\frac{4}{5}-u\right) \pi\right)+\frac{1}{2}, & u \in\left[0, \frac{4}{5}\right], \\ \cos ^{2}\left(\left(\frac{4}{5}-u\right) \pi\right)+\frac{1}{2}+200 \arctan \left(u-\frac{4}{5}\right), & u \in\left[\frac{4}{5}, \frac{7}{5}\right], \\ \cos ^{2}\left(\left(\frac{4}{5}-u\right) \pi\right)+\frac{1}{2}+200 \arctan \left(u-\frac{4}{5}\right) & \\ \quad+\frac{1}{3} \sin ^{2}\left(u-\frac{7}{5}\right), & u \in\left[\frac{7}{5}, \infty\right) .\end{cases}
$$

Set $m=3, \alpha=3 / 2, \eta=5 / 2, k=6, a(t)=e^{-t}, \lambda_{1}=1 / 10, \lambda_{2}=\pi, \lambda_{3}=1 / 3, \beta_{1}=3 / 4, \beta_{2}=5 / 2$, $\beta_{3}=7 / 2$, and we can obtain

$$
\Omega=\Gamma(\alpha)-\sum_{i=1}^{m} \frac{\lambda_{i} \Gamma(\alpha)}{\Gamma\left(\alpha+\beta_{i}\right)}(\log \eta)^{\alpha+\beta_{i}-1} \approx 0.450449
$$

By direct calculation we can get

$$
\begin{aligned}
& M=\left(\frac{1}{\Gamma(\alpha)}+\sum_{i=1}^{m} \frac{\lambda_{i}(\log \eta)^{\alpha+\beta_{i}-1}}{\Omega \Gamma\left(\alpha+\beta_{i}\right)}\right) \int_{1}^{\infty} a(s) \frac{d s}{s} \approx 0.487034 \\
& m=\sum_{i=1}^{m} \frac{\lambda_{i}(\log \eta)^{2 \alpha+\beta_{i}-2}}{\Omega \Gamma\left(\alpha+\beta_{i}\right)\left(1+(\log \eta)^{\alpha-1}\right)} \int_{\eta}^{k \eta} a(s) \frac{d s}{s} \approx 0.013302 .
\end{aligned}
$$

Choose $a=4 / 5, b=7 / 5$, and $c=160$; then $f$ satisfies

$$
\begin{aligned}
& f\left(\left(1+(\log t)^{\frac{1}{2}}\right) u\right) \leq 1.5<1.6426 \approx \frac{a}{M}, \quad(t, u) \in[1, \infty) \times\left[0, \frac{4}{5}\right], \\
& f\left(\left(1+(\log t)^{\frac{1}{2}}\right) u\right) \geq 219.4760>105.2492 \approx \frac{b}{m}, \quad(t, u) \in\left[\frac{5}{2}, 15\right] \times\left[\frac{7}{5}, 160\right], \\
& f\left(\left(1+(\log t)^{\frac{1}{2}}\right) u\right) \leq 315.9926<328.5189 \approx \frac{c}{M}, \quad(t, u) \in[1, \infty) \times[0,160] .
\end{aligned}
$$

Thus, by an application of Theorem 3.3 the boundary value problem (5.1) has at least three positive solutions $u_{1}, u_{2}, u_{3}$ such that

$$
\sup _{t \in[1, \infty)} \frac{\left|u_{1}(t)\right|}{1+(\log t)^{\frac{1}{2}}}<\frac{4}{5}, \quad \frac{7}{5}<\min _{t \in\left[\frac{5}{2}, 15\right]} \frac{u_{2}(t)}{1+(\log t)^{\frac{1}{2}}}
$$

and

$$
\frac{4}{5}<\sup _{t \in[1, \infty)} \frac{\left|u_{3}(t)\right|}{1+(\log t)^{\frac{1}{2}}} \quad \text { with } \min _{t \in\left[\frac{5}{2}, 15\right]} \frac{u_{3}(t)}{1+(\log t)^{\frac{1}{2}}}<\frac{7}{5} .
$$


Example 5.2 We consider the following Hadamard fractional differential equation with nonlocal boundary conditions on unbounded domain:

$$
\left\{\begin{array}{l}
D^{\frac{4}{3}} u(t)+t^{-2} f(u(t))=0, \quad t \in(1, \infty), \\
u(1)=0, \quad D^{\frac{1}{3}} u(\infty)=2 I^{\sqrt{\pi}} u(3)+e^{-1} I^{\frac{1}{2}} u(3)+\sin (11) I^{\frac{9}{7}} u(3)+\frac{\sqrt{\pi}}{2} I^{\frac{11}{3}} u(3),
\end{array}\right.
$$

where

$$
f(u(t))= \begin{cases}2 e^{-u}+\frac{1}{5} \cos ^{2}\left(\frac{u \pi}{2}\right)+22, & u \in[0,2], \\ 2 e^{-u}+\frac{1}{5} \cos ^{2}\left(\frac{u \pi}{2}\right)+19+\frac{8}{\pi} \arctan (u-2) & \\ +3 \sin ^{2}\left(\frac{(u+1) \pi}{6}\right), & u \in[2, \infty) .\end{cases}
$$

Set $m=4, \alpha=4 / 3, \eta=3, k=4, a(t)=t^{-2}, \lambda_{1}=2, \lambda_{2}=e^{-1}, \lambda_{3}=\sin (11), \lambda_{4}=\sqrt{\pi} / 2$, $\beta_{1}=\sqrt{\pi}, \beta_{2}=1 / 2, \beta_{3}=9 / 7, \beta_{4}=11 / 3$, and we can obtain

$$
\Omega=\Gamma(\alpha)-\sum_{i=1}^{m} \frac{\lambda_{i} \Gamma(\alpha)}{\Gamma\left(\alpha+\beta_{i}\right)}(\log \eta)^{\alpha+\beta_{i}-1} \approx 0.199034
$$

By direct calculation we can get

$$
\begin{aligned}
& M=\left(\frac{1}{\Gamma(\alpha)}+\sum_{i=1}^{m} \frac{\lambda_{i}(\log \eta)^{\alpha+\beta_{i}-1}}{\Omega \Gamma\left(\alpha+\beta_{i}\right)}\right) \int_{1}^{\infty} a(s) \frac{d s}{s} \approx 2.512136, \\
& m=\sum_{i=1}^{m} \frac{\lambda_{i}(\log \eta)^{2 \alpha+\beta_{i}-2}}{\Omega \Gamma\left(\alpha+\beta_{i}\right)\left(1+(\log \eta)^{\alpha-1}\right)} \int_{\eta}^{k \eta} a(s) \frac{d s}{s} \approx 0.103271 .
\end{aligned}
$$

Choose $r_{1}=2, r_{2}=100, \rho_{1}=10$, and $\rho_{2}=3 / 10$; then $f$ satisfies

$$
\begin{aligned}
& f\left(\left(1+(\log t)^{\frac{1}{3}}\right) u\right) \geq 22 \geq 20=\rho_{1} r_{1}, \quad(t, u) \in[1, \infty) \times[0,2], \\
& f\left(\left(1+(\log t)^{\frac{1}{3}}\right) u\right) \leq 28.2 \leq 30=\rho_{2} r_{2}, \quad(t, u) \in[1, \infty) \times[0,100] .
\end{aligned}
$$

Thus, by an application of Theorem 4.2 the boundary value problem (5.2) has at least one positive solution $u$ such that

$$
2 \leq \sup _{t \in[1, \infty)} \frac{|u(t)|}{1+(\log t)^{\frac{1}{3}}} \leq 100
$$

\section{Conclusions}

We have investigated the existence of nonnegative multiple solutions for nonlinear fractional differential equations of Hadamard type, with nonlocal fractional integral boundary conditions on an unbounded domain. The existence of at least one positive solution is proved by applying Guo-Krasnoselskii's fixed point theorem, while the existence of at least three distinct nonnegative solutions is established by means of the well-known LeggettWilliams fixed point theorem. For our study a special Banach space was introduced. The results are well illustrated with the aid of examples. The results reported in this study significantly contribute to the study of positive solutions for Hadamard fractional differential equations on infinite intervals. 


\section{Competing interests}

The authors declare that they have no competing interests.

\section{Authors' contributions}

All authors contributed equally in this article. They read and approved the final manuscript.

\section{Author details}

${ }^{1}$ Nonlinear Dynamic Analysis Research Center, Department of Mathematics, Faculty of Applied Science, King Mongkut's University of Technology North Bangkok, Bangkok, 10800, Thailand. '2Department of Mathematics, University of loannina, Ioannina, 451 10, Greece. ${ }^{3}$ Nonlinear Analysis and Applied Mathematics (NAAM) - Research Group, Department of Mathematics, Faculty of Science, King Abdulaziz University, P.O. Box 80203, Jeddah, 21589, Saudi Arabia. ${ }^{4}$ Centre of Excellence in Mathematics, CHE, Sri Ayutthaya Rd., Bangkok, 10400, Thailand.

\section{Acknowledgements}

This research is partially supported by the Centre of Excellence in Mathematics, the Commission on Higher Education, Thailand.

\section{Received: 2 November 2015 Accepted: 14 March 2016 Published online: 22 March 2016}

\section{References}

1. Kilbas, AA, Srivastava, HM, Trujillo, JJ: Theory and Applications of Fractional Differential Equations. North-Holland Mathematics Studies, vol. 204. Elsevier, Amsterdam (2006)

2. Diethelm, K: The Analysis of Fractional Differential Equations. Springer, Berlin (2010)

3. Samko, SG, Kilbas, AA, Marichev, Ol: Fractional Integrals and Derivatives: Theory and Applications. Gordon \& Breach, Yverdon (1993)

4. Podlubny, I: Fractional Differential Equations. Academic Press, San Diego (1999)

5. Agarwal, RP, Zhou, Y, He, Y: Existence of fractional neutral functional differential equations. Comput. Math. Appl. 59, 1095-1100 (2010)

6. Ahmad, B, Nieto, JJ: Boundary value problems for a class of sequential integrodifferential equations of fractional order. J. Funct. Spaces Appl. 2013, Article ID 149659 (2013)

7. Ahmad, B, Ntouyas, SK, Alsaedi, A: A study of nonlinear fractional differential equations of arbitrary order with Riemann-Liouville type multistrip boundary conditions. Math. Probl. Eng. 2013, Article ID 320415 (2013)

8. Ahmad, B, Ntouyas, SK: Nonlocal fractional boundary value problems with slit-strips integral boundary conditions. Fract. Calc. Appl. Anal. 18, 261-280 (2015)

9. Baleanu, D, Diethelm, K, Scalas, E, Trujillo, JJ: Fractional Calculus Models and Numerical Methods. Series on Complexity, Nonlinearity and Chaos. World Scientific, Boston (2012)

10. Baleanu, D, Mustafa, OG, Agarwal, RP: On $L^{P}$-solutions for a class of sequential fractional differential equations. Appl. Math. Comput. 218, 2074-2081 (2011)

11. Hadamard, J: Essai sur l'étude des fonctions données par leur développment de Taylor. J. Math. Pures Appl. 8, 101-186 (1892)

12. Butzer, PL, Kilbas, AA, Trujillo, JJ: Compositions of Hadamard-type fractional integration operators and the semigroup property. J. Math. Anal. Appl. 269, 387-400 (2002)

13. Butzer, PL, Kilbas, AA, Trujillo, JJ: Fractional calculus in the Mellin setting and Hadamard-type fractional integrals. J. Math. Anal. Appl. 269, 1-27 (2002)

14. Butzer, PL, Kilbas, AA, Trujillo, JJ: Mellin transform analysis and integration by parts for Hadamard-type fractional integrals. J. Math. Anal. Appl. 270, 1-15 (2002)

15. Wang, JR, Zhang, Y: On the concept and existence of solutions for fractional impulsive systems with Hadamard derivatives. Appl. Math. Lett. 39, 85-90 (2015)

16. Yukunthorn, W, Ahmad, B, Ntouyas, SK, Tariboon, J: On Caputo-Hadamard type fractional impulsive hybrid systems with nonlinear fractional integral conditions. Nonlinear Anal. Hybrid Syst. 19, 77-92 (2016)

17. Kilbas, AA: Hadamard-type fractional calculus. J. Korean Math. Soc. 38, 1191-1204 (2001)

18. Kilbas, AA, Trujillo, JJ: Hadamard-type integrals as G-transforms. Integral Transforms Spec. Funct. 14, $413-427$ (2003)

19. Agarwal, RP, O'Regan, D: Infinite Interval Problems for Differential, Difference and Integral Equations. Kluwer Academic, Dordrecht (2001)

20. O'Regan, D: Theory of Singular Boundary Value Problems. World Scientific, River Edge (1994)

21. Liu, Y: Existence and unboundedness of positive solutions for singular boundary value problems on half-line. Appl. Math. Comput. 144, 543-556 (2003)

22. Liang, S, Zhang, J: Existence of multiple positive solutions for $m$-point fractional boundary value problems on an infinite interval. Math. Comput. Model. 54, 1334-1346 (2011)

23. Liu, YS: Boundary value problem for second order differential equations on unbounded domain. Acta Anal. Funct. Appl. 4, 211-216 (2002)

24. Zhang, L, Ahmad, B, Wang, G, Agarwal, R, Al-Yami, M, Shammakh, W: Nonlocal integrodifferential boundary value problem for nonlinear fractional differential equations on an unbounded domain. Abstr. Appl. Anal. 2013, Article ID $813903(2013)$

25. Zhao, X, Ge, W: Unbounded solutions for a fractional boundary value problems on the infinite interval. Acta Appl. Math. 109, 495-505 (2010)

26. Thiramanus, P, Ntouyas, SK, Tariboon, J: Existence of solutions for Riemann-Liouville fractional differential equations with nonlocal Erdélyi-Kober integral boundary conditions on the half-line. Bound. Value Probl. 2015, 196 (2015)

27. Leggett, RW, Williams, LR: A fixed point theorem with application to an infectious disease model. J. Math. Anal. Appl. 76, 91-97 (1980)

28. Guo, D, Lakshmikantham, V: Nonlinear Problems in Abstract Cones. Academic Press, San Diego (1988) 